\title{
Kinetics and thermodynamics of sorption of nitroaromatic compounds to as-grown and oxidized multiwalled carbon nanotubes
}

\author{
Xiu-E Shen ${ }^{\mathrm{a}, \mathrm{b}}$, Xiao-Quan Shan ${ }^{\mathrm{b}, *}$, De-Ming Dong ${ }^{\mathrm{a}, *}$, Xiu-Yi Hua $^{\mathrm{a}}$, Gary Owens ${ }^{\mathrm{c}}$ \\ a College of Environment and Resources, Jilin University, Changchun 130012, China

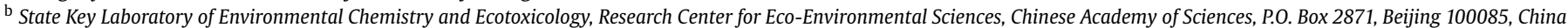 \\ c Centre for Environmental Risk Assessment and Remediation, University of South Australia, Mawson Lakes, SA 5095, Australia
}

\section{A R T I C L E I N F O}

Article history:

Received 19 May 2008

Accepted 11 October 2008

Available online 1 November 2008

\section{Keywords:}

Nitroaromatic compounds

Multiwalled carbon nanotubes

Nitric acid oxidization

Sorption kinetics

Sorption thermodynamics

\begin{abstract}
A B S T R A C T
The sorption kinetics and thermodynamics of 1,3-dinitrobenzene (DNB), $m$-nitrotoluene (mNT), $p$-nitrophenol (pNP), and nitrobenzene (NB) on as-grown and nitric acid-oxidized multiwalled carbon nanotubes (MWCNTs) were investigated. The sorption kinetics was well described by a pseudo-second-order rate model, while both Langmuir and Freundlich models described the sorption isotherms well and the sorption thermodynamic parameters of equilibrium constant $\left(K_{0}\right)$, standard free energy $(\Delta G)$, standard enthalpy $(\Delta H)$, and standard entropy changes $(\Delta S)$ were measured. The values of $\Delta H$ and $\Delta G$ suggested that the sorption of nitroaromatics (NACs) onto MWCNTs was exothermic and spontaneous. The structure, number, and position of nitro groups of NACs were the main factors affecting the sorption rate and capacity. Treatment of the MWCNTs with nitric acid increased both the surface area and the pore volume and introduced oxygen-containing functional groups to the MWCNTs, which depressed the sorption of NACs onto MWCNTs.
\end{abstract}

(c) 2008 Elsevier Inc. All rights reserved.

\section{Introduction}

Carbon nanotubes (CNTs) have unique physicochemical and electrical properties and have widespread applications in field emission, in hydrogen storage, and as chemical sensors. Due to their high surface area and large micropore volume, CNTs are also considered to be extremely good adsorbents and since the first report of their successful removal of dioxins [1], CNTs have been utilized for the sorption of a large number of different organic compounds from water [2-8]. The sorption properties of CNTs can be modified by chemical oxidation treatments using sodium hypochlorite $(\mathrm{NaOCl})$, hydrogen peroxide $\left(\mathrm{H}_{2} \mathrm{O}_{2}\right)$, potassium permanganate $\left(\mathrm{KMnO}_{4}\right)$, and nitric acid $\left(\mathrm{HNO}_{3}\right)[2,5]$. Such oxidation treatments are able to remove impurities and hemispherical caps, increasing the surface area and introducing oxygen-containing functional groups $[6,9]$, hence altering the sorption properties for organic chemicals $[2,3,5]$. For example, following treatment with acid, CNTs become more suitable for the sorption of low molecular weight and relatively polar trihalomethane molecules [3]. In another study, oxidation with nitric acid increased the sorption of $p$-xylene, while decreasing the sorption of $o$-xylene on singlewalled carbon nanotubes (SWCNTs), suggesting that the sorption

\footnotetext{
* Corresponding authors. Fax: +86 1062923563.

E-mail addresses: xiaoquan@rcees.ac.cn (X.-Q. Shan),dmdong@mail.jlu.edu.cn (D.-M. Dong).
}

of $o$-xylene and $p$-xylene onto SWCNTs was mainly influenced by the positions of the methyl groups on xylene molecules and the presence of oxygen-containing groups on the surface of SWCNTs [5]. However, a recent study [8] revealed that incorporated surface oxides on CNTs created polar regions that reduced the surface area available for naphthalene sorption, and that as little as a $10 \%$ increase in surface oxygen concentration resulted in a $70 \%$ decrease in the maximum sorption capacity of naphthalene. This study indicated that oxidative treatments may not always beneficially enhance sorption of organic compounds and pointed to the need to investigate sorption of specific compounds on a case by case basis.

Nitroaromatic compounds (NACs) are widely used as pesticides, explosives, and intermediates in the synthesis of dyes and other high-volume chemicals. NACs are highly polar and often act as strong electron acceptors when interacting with adsorbentcontaining structures with high electron polarization or with electron donors such as oxygen-containing groups. It was recently reported [7] that CNTs have a stronger affinity for NACs than for other nonpolar chemicals. This was attributed to the formation of an electron donor-acceptor (EDA) complex between NACs (electron acceptor) and the graphene sheets of the carbon nanotubes (electron donor). Sorption properties of NACs on activated carbon have been studied for many years and involve electrostatic and dispersive interactions between the adsorbate and the activated carbon $[10,11]$. Electrostatic interactions dominate when the adsorbate is dissociated under the experimental conditions, while three mecha- 
Table 1

Selected properties of NACs used in this study.

\begin{tabular}{llllcll}
\hline NACs & $\begin{array}{l}\text { MW } \\
(\mathrm{g} / \mathrm{mol})\end{array}$ & $\log K_{\text {ow }}$ & $\mathrm{p} K_{\mathrm{a}}$ & $\begin{array}{l}C_{\mathrm{s}}\left(25^{\circ} \mathrm{C}\right) \\
(\mathrm{mg} / \mathrm{L})\end{array}$ & $\begin{array}{l}V_{\mathrm{s}} \\
\left(\mathrm{cm}^{3} / \mathrm{mol}\right)\end{array}$ & $\begin{array}{l}S_{\mathrm{Vdw}} \\
\left(\AA^{2}\right)\end{array}$ \\
\hline DNB & 168.1 & 1.49 & - & 533 & 106.5 & 85.26 \\
$\mathrm{mNT}$ & 137.1 & 2.42 & - & 498 & 103.2 & 78.21 \\
$\mathrm{pNP}$ & 139.1 & 2.04 & 7.15 & 16000 & 94.9 & 76.61 \\
NB & 123.1 & 1.84 & - & 1900 & 89.1 & 71.92 \\
\hline
\end{tabular}

a MW, molecular weight; $K_{\mathrm{ow}}$, octanol-water partition coefficient; $C_{\mathrm{s}}$, water solubility; $V_{\mathrm{s}}$, molar volume; $S_{\mathrm{Vdw}}$, molecular Van der Waals square.

nisms are proposed for nonelectrostatic interactions, (1) $\pi-\pi$ dispersion interaction, (2) hydrogen bonding [12], and (3) electron donor-acceptor complex formation [13]. Of theses the $\pi-\pi$ dispersion interaction mechanism is the most widely accepted [11, 14]. For the sorption of nitrobenzene onto activated carbon, which is undissociated in solution, the dispersive interactions will be the most important mechanism in the sorption process [15]. Numerous studies have also been conducted on the specific interaction between NACs and phyllosilicate minerals such as clays to which NACs may adsorb strongly and reversibly. Specific sorption to clays was believed to occur via electron donor-acceptor complex formation with oxygen present at the external siloxane surface of the clay minerals $[16,17]$. Since oxidation of CNTs introduces oxygencontaining functional groups it is possible that this would enhance sorption of organic compounds. However, the exact role of these oxygen-containing functional groups on the specific sorption of NACs on CNTs still needs to be determined.

Understanding the kinetics and thermodynamics of the sorption process is critical for the development of more efficient sorbents suitable for real world applications. While studies of the sorption kinetics and thermodynamics of metals are generally well known, very little information is currently available regarding the sorption of organic compounds, such as trihalomethanes [18] and dyes [19], onto MWCNTs. These limited studies suggested that the sorption of the reactive dye and trihalomethanes was spontaneous and mainly due to physical sorption. In this work, the sorption kinetics and thermodynamics of NACs on CNTs were systematically studied for the first time.

The aim of this work was to understand the interaction between NACs and MWCNTs, and to reveal the effect of oxygencontaining functional groups on the sorption of NACs to MWCNTs.

\section{Materials and methods}

\subsection{Materials}

MWCNTs were purchased from Tsinghua University, and were prepared by dissociating methane in a hydrogen flow at $900 \mathrm{~K}$ using Ni nanoparticles supported on diatomite as a catalyst. Asgrown MWCNTs $(2 \mathrm{~g})$ were added to $3 \mathrm{M} \mathrm{HNO}_{3}(400 \mathrm{~mL})$ with ultrasonic stirring for $24 \mathrm{~h}$ to dissolve the catalyst support and $\mathrm{Ni}$ particles in the MWCNTs. The suspension was then filtered and rinsed with deionized water until the $\mathrm{pH}$ of the final suspension reached about 6 before drying at $80^{\circ} \mathrm{C}$. The oxidized MWCNTs were subsequently calcined at $450^{\circ} \mathrm{C}$ for $24 \mathrm{~h}$ to remove amorphous carbon.

1,3-Dinitrobenzene (DNB), $m$-nitrotoluene ( $\mathrm{mNT}$ ), $p$-nitrophenol (pNP), and nitrobenzene (NB) were purchased from Beijing Chemicals (Beijing, China). No significant impurities were detected by HPLC-UV analysis. Selected properties of DNB, mNT, pNP, and NB are listed in Table 1 . The adsorbates were dissolved in $0.01 \mathrm{M}$ $\mathrm{NaNO}_{3}$ and $100 \mathrm{mg} / \mathrm{L} \mathrm{NaN}_{3}$ solution which was used as support electrolyte throughout this study. The final concentrations were limited to $<50 \%$ of their water solubility to ensure complete dissolution.
Table 2

Selected properties of MWCNTs.

\begin{tabular}{llllll}
\hline MWCNTs & $\begin{array}{l}\text { BET surface } \\
\text { area } \\
\left(\mathrm{m}^{2} / \mathrm{g}\right)\end{array}$ & $\begin{array}{l}\text { Average pore } \\
\text { diameter } \\
(\mathrm{nm})\end{array}$ & $\begin{array}{l}\text { Pore } \\
\text { volume } \\
\left(\mathrm{cm}^{3} / \mathrm{g}\right)\end{array}$ & $\begin{array}{l}\text { Carbon } \\
\text { content } \\
(\%)\end{array}$ & $\begin{array}{l}\text { Hydrogen } \\
\text { content } \\
(\%)\end{array}$ \\
\hline As grown & 159.7 & 9.57 & 0.382 & 93.0 & 0.26 \\
Oxidized & 274.0 & 9.52 & 0.652 & 90.0 & 0.19 \\
\hline
\end{tabular}

\subsection{Characterization of MWCNTs}

The morphology of MWCNTs was characterized by high-resolution transmission electron microscope (HRTEM, JEOL JEM-2010, Tokyo, Japan). The surface area and pore volume were measured by nitrogen gas adsorption and desorption at $77 \mathrm{~K}$ with ASAP2000 (Micromeritics Instrument Corporation). The multipoint BrunauerEmmett-Teller method was used to calculate the surface area. The Barrett-Joyner-Halenda method was used to calculate the mesopore volumes. The functional groups on the surface of MWCNTs were detected by a Fourier transform infrared (FTIR) spectroscopy equipped with deuterated triglycine (DTGS) and mercurycadmium-telluride (MCT) detectors (NEXUS 670 FTIR, Nicolet, USA). The characteristics of MWCNTs are listed in Table 2.

\subsection{Kinetic experiments}

Kinetic experiments were performed in 40 - $\mathrm{mL}$ glass centrifuge tubes sealed with Teflon-lined screw caps at $20.0 \pm 0.5^{\circ} \mathrm{C}$. Initially MWCNTs (10 mg) were introduced to the supporting solution of $0.01 \mathrm{~mol} / \mathrm{L} \mathrm{NaNO}_{3}$ containing $100 \mathrm{mg} / \mathrm{L} \mathrm{NaN}_{3}$ solution $(30 \mathrm{~mL})$ and prior to the introduction of NACs, the tubes were rotated for $1.5 \mathrm{~h}$ to hydrate the MWCNTs. The initial concentration was $12 \mathrm{mg} / \mathrm{L}$ for each NAC of interest. After the introduction of the NACs, samples were shaken for a certain time intervals, filtered through $0.45-\mu \mathrm{m}$ cellulose acetate membrane filter, and analyzed for the equilibrium solution concentration of NAC. The quantity of NACs sorbed at time $t(\mathrm{~min}), q_{\mathrm{t}}(\mathrm{mg} / \mathrm{g})$, was deduced from the mass difference between initial $(t=0 \mathrm{~min})$ and final solution concentration taken at time $t$ (min).

\subsection{Batch sorption experiments}

Batch-equilibration sorption experiments were carried out in triplicate by mixing MWCNTs $\left(10 \mathrm{mg}\right.$ ) and $0.01 \mathrm{~mol} / \mathrm{L} \mathrm{NaNO}_{3}$ and $100 \mathrm{mg} / \mathrm{L} \mathrm{NaN}_{3}$ solution $(30 \mathrm{~mL}$ ) containing various concentrations of adsorbates in $40-\mathrm{mL}$ glass centrifuge tubes sealed with Teflonlined screw caps. Initial concentrations of NACs were chosen so as to control the amount of NACs adsorbed to be between 20 and $80 \%$ of the total initial concentration. The tubes were shaken in the dark on a rotary shaker at $100 \mathrm{rpm}$ for $24 \mathrm{~h}$ at $10 \pm 1,20 \pm 1$, and $35 \pm 1{ }^{\circ} \mathrm{C}$. After rotation, all the tubes were placed vertically on a flat surface in the dark for $24 \mathrm{~h}$ at the set temperature to ensure separation between solution and MWCNTs. Centrifugation was not used to avoid any temperature change. The concentrations of NACs in the supernatants were analyzed by direct injection of supernatants to HPLC (Agilent Technologies, Wilmington, CT). Blank experiments were performed using the same experimental procedure but without added MWCNTs to check for potential sorption of NACs to the glass centrifuge tubes or the membrane filters, which could potentially result in nonspecific loss and or degradation of the NACs. However, such losses in the blank experiments were less than $5 \%$ of the initial concentrations and therefore indicated that since losses were small, the amount of NACs sorbed by MWCNTs could be calculated directly from mass difference between the initial and final equilibrium concentrations.

Control of the $\mathrm{pH}$ of the solution suspensions was not considered critical for $\mathrm{DNB}, \mathrm{mNT}$, and $\mathrm{NB}$ because all the adsorbates were 
nondissociable, but for pNP, the $\mathrm{pH}$ was controlled to $6.0 \pm 0.5$ by the dropwise addition of $\mathrm{NaOH}$ and $\mathrm{HNO}_{3}$ as required. The final $\mathrm{pH}$ of the supernatant was measured at the end of the equilibration and was shown to be identical to the initial concentration, indicating that the $\mathrm{pH}$ remained constant at $6.0 \pm 0.5$.

\subsection{Data analysis}

For kinetic analysis, four kinetic models including (1) pseudosecond-order model, (2) parabolic diffusion model, (3) modified Freundlich model, and (4) Elovich model were tested for goodness of fit of the experimental data using the coefficient of correlation $\left(R^{2}\right)$ as a measure of the agreement between the four proposed models and the experimental data. Each of the models is discussed briefly below.

\subsubsection{Pseudo-second-order model}

This model includes all the steps of sorption including external film diffusion, adsorption, and internal particle diffusion [20-22]. This model assumes that the differences between the average solid phase concentration at time $t(\mathrm{~min}), q_{\mathrm{t}}(\mathrm{mg} / \mathrm{g})$, and the equilibrium concentration, $q_{\mathrm{e}}(\mathrm{mg} / \mathrm{g})$, are the driving force for adsorption and the overall adsorption rate is proportional to the square of the driving force, which can be written as [23]

$\frac{d q_{\mathrm{t}}}{d t}=k_{2}\left(q_{\mathrm{e}}-q_{\mathrm{t}}\right)^{2}$

where $k_{2}[\mathrm{~g} /(\mathrm{mg} \mathrm{min})]$ is the rate constant of the pseudo-secondorder adsorption, and is a complex function of the initial concentration of solute [24], and $q_{\mathrm{e}}$ and $q_{\mathrm{t}}$ are the adsorbed NACs $(\mathrm{mg} / \mathrm{g}$ ) at equilibrium and at time $t(\mathrm{~min})$. Given that the initial sorption rate $v_{0}[\mathrm{mg} /(\mathrm{g} \min )]$ is

$v_{0}=k_{2} q_{\mathrm{e}}^{2}$,

Eq. (1) can be rearranged to give the linearized expression:

$\frac{t}{q_{\mathrm{t}}}=\frac{1}{v_{0}}+\frac{1}{q_{\mathrm{e}}} t$.

Thus the values of $v_{0}$ and $k_{2}$ can be determined experimentally by plotting $t / q_{\mathrm{t}}$ versus $t$ and extracting information from the least-squares analysis of slope and intercept and substituting into Eq. (2).

\subsubsection{Parabolic diffusion model}

The parabolic diffusion model can be used to determine whether diffusion-controlled phenomena are a rate-limiting step during sorption [25]. The parabolic diffusion model can be written as

$\frac{q_{\mathrm{t}}}{q_{\mathrm{e}} t}=k t^{-0.5}+a$,

where $q_{\mathrm{e}}$ and $q_{\mathrm{t}}$ are the adsorbed NACs ( $\mathrm{mg} / \mathrm{g}$ ) at equilibrium and at time $t(\mathrm{~min}), a$ is a constant, and $k$ is the overall diffusion constant for sorption.

\subsubsection{Modified Freundlich model}

The modified Freundlich model can be written as [25]

$q_{\mathrm{t}}=k C_{0} t^{a}$,

where $q_{\mathrm{t}}$ is the adsorbed NACs $(\mathrm{mg} / \mathrm{g})$ at time $t(\mathrm{~min}), k$ is the rate constant for the sorption, $a$ is a constant, and $C_{0}$ is the initial concentration of the solute.

\subsubsection{Elovich model}

The Elovich model can be written as [25]

$q_{\mathrm{t}}=a \ln (t)+b$,

where $q_{\mathrm{t}}$ is the adsorbed NACs $(\mathrm{mg} / \mathrm{g})$ at time $t$ (min), and $a$ and $b$ are constants whose chemical significance is not clearly defined.

For isotherm modeling, Langmuir and Freundlich equations are the two most commonly applied models suitable for representing the nonlinear adsorption of hydrophobic organic chemicals to activated carbon, charcoal, and carbon nanotubes. In this study, both the Langmuir and the Freundlich models were employed to fit the experimental data. The Langmuir equation is

$q_{\mathrm{e}}=\frac{Q_{\mathrm{m}} C_{\mathrm{e}}}{K_{\mathrm{L}}+C_{\mathrm{e}}}$

where $q_{\mathrm{e}}(\mathrm{mg} / \mathrm{g})$ is the equilibrium sorbed concentration, $C_{\mathrm{e}}$ $(\mathrm{mg} / \mathrm{L})$ is the equilibrium solution phase concentration, $K_{\mathrm{L}}(\mathrm{mg} / \mathrm{L})$ is the affinity parameter, and $Q_{m}(\mathrm{mg} / \mathrm{g})$ is the maximum sorption capacity. The Freundlich model is an empirical equation that assumes heterogeneous adsorption due to the diversity of adsorption sites,

$q_{\mathrm{e}}=K_{\mathrm{F}} C_{\mathrm{e}}^{1 / n}$,

where $q_{\mathrm{e}}(\mathrm{mg} / \mathrm{g})$ is the equilibrium sorbed concentration, $C_{\mathrm{e}}$ $(\mathrm{mg} / \mathrm{L})$ is the equilibrium solution phase concentration, $K_{\mathrm{F}}$ is the Freundlich adsorption coefficient $\left[(\mathrm{mg} / \mathrm{g})(\mathrm{L} / \mathrm{mg})^{1 / n}\right]$, and $1 / n$ is a constant indicating the isotherm curvature.

The isotherms can be expressed either on a mass or on a surface area basis, and in this study, both forms were used. The use of surface area normalized isotherms was necessary to compare the uptake of NACs by nitric acid-oxidized MWCNTs with that of as-grown MWCNTs having different surface areas and pore size distributions.

\section{Results and discussion}

\subsection{Characterization of as-grown and nitric-oxidized MWCNTS}

Figs. 1a, 1b, 1c, and 1d are TEM images of the MWCNTs before and after oxidation with nitric acid. Prior to oxidation TEM images of the MWCNTs showed a large amount of impure particles, such as amorphous carbon and metal particles (Figs. 1a and $1 \mathrm{~b})$. While after oxidation the MWCNTs bundles had very little visible attachment of particles, indicating that oxidization had successfully removed these impurities (Figs. 1c and 1d). Due to strong van der Waals interactions, MWCNTs adhere to each other and form bundles and the space between the bundles can be considered as pores as shown in Figs. 1a and 1c. Agnihotri et al. [26] identified different adsorption sites on a bundle of CNTs. For an individual carbon nanotube, the intratube and the external graphene surface are the main sorption sites. However, the process of forming bundles or pores creates new adsorption sites, such as the interstitial channel, the external groove, and partial coating of the external surface by nanometer-thick layered carbon. All of these sites contribute to overall adsorption and may also include adsorption due to some carbon-coated catalyst particles. Table 2 lists the physical properties of as-grown and oxidized MWCNTs. The BET surface area $\left(274.0 \mathrm{~m}^{2} / \mathrm{g}\right)$ and the pore volume $\left(0.652 \mathrm{~cm}^{3} / \mathrm{g}\right)$ of oxidized MWCNTs were greater than those of as-grown MWCNTs $\left(159.7 \mathrm{~m}^{2} / \mathrm{g}\right.$ and $0.382 \mathrm{~cm}^{3} / \mathrm{g}$ ), but the average pore diameter of the two carbon nanotubes were approximately the same (Table 2). The increase of the BET surface area and pore volume after oxidization was attributed to removal of amorphous carbon and hemispherical caps on the nanotubes 

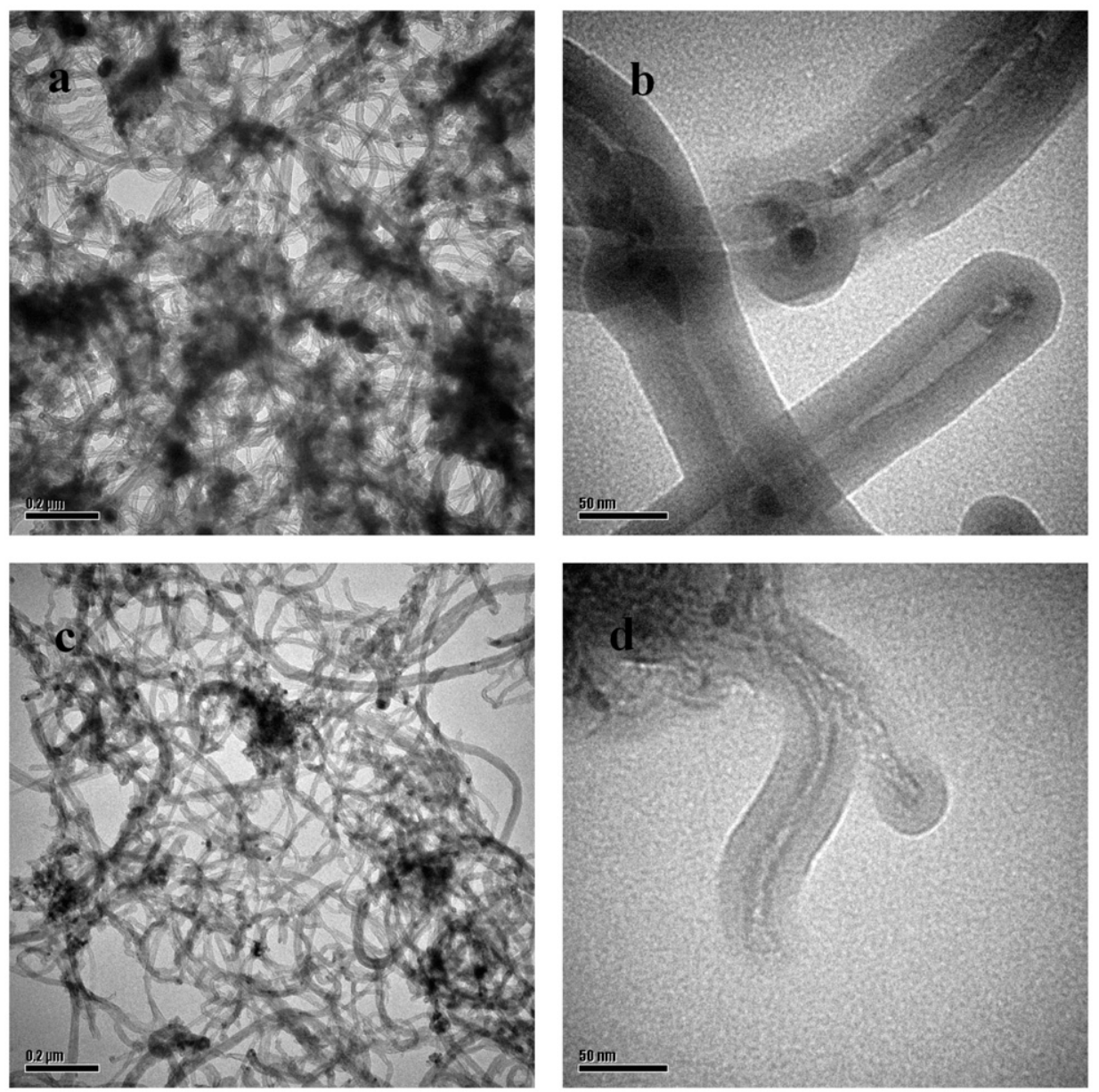

Fig. 1. TEM images of MWCNTs: (a and b) as-grown MWCNTs; (c and d) nitric acid-oxidized MWCNTs.

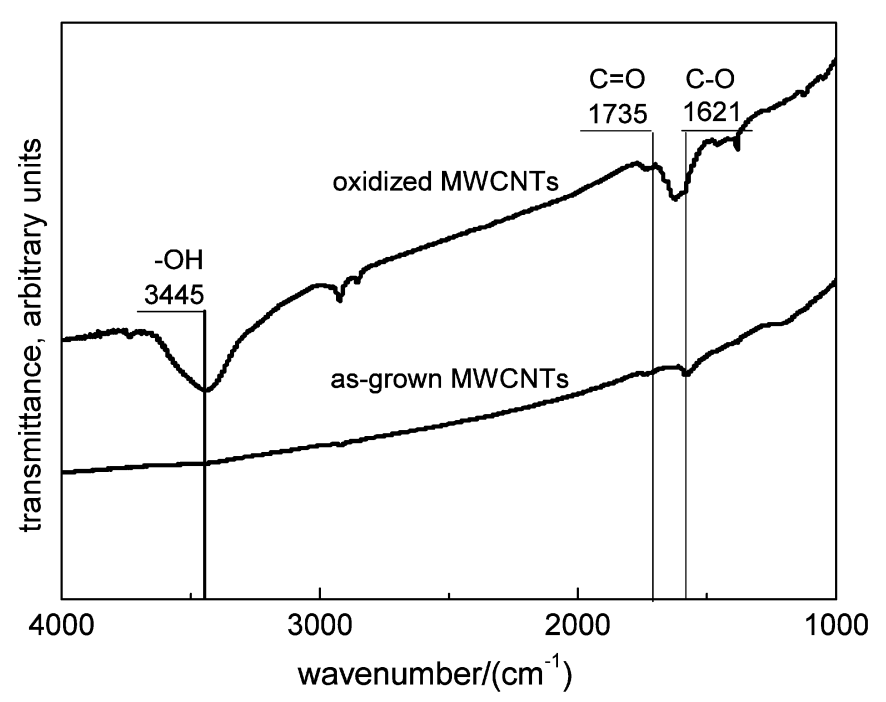

Fig. 2. FTIR spectra of as-grown and oxidized MWCNTs.

(Fig. 1d). Compared to the as-grown MWCNTs, the FTIR spectrum of the oxidized MWCNTs confirmed the existence of the carboxylic groups $(-\mathrm{COOH})$, carbonyl groups $(-\mathrm{CO})$, and hydroxyl groups $(-\mathrm{OH})$ (Fig. 2). All of the above results showed that after oxidization the carbon nanotubes became more hydrophilic and had more active functional groups on the surface while the physical properties such as specific surface area and pore size distribution were increased.

\subsection{Sorption kinetics of NACS}

Fig. 3 shows the kinetics of NACs sorption onto the as-grown and nitric acid-oxidized MWCNTs. The plots represented the adsorbed NACs onto the MWCNTs $\left(q_{\mathrm{t}}\right)$ versus time at an initial concentration of $12 \mathrm{mg} / \mathrm{L}$. For all the NACs considered, the sorption was very fast on both as-grown and nitric acid-oxidized MWCNTs. Apparent equilibrium was reached within $180 \mathrm{~min}$, for most of the NACs considered within $30 \mathrm{~min}$ and for NB, sorption occurred within $10 \mathrm{~min}$. This fast sorption process was similar to that which had been observed for trihalomethanes [18] and reactive dyes [19]. Individual MWCNTs have a dominant adsorption domain which is primarily the outermost graphene surface and defect sites. However, when MWCNTs form bundles in aqueous media, the interstitial channel space between the tubes within the bundles can be regarded as pores. NACs were thought to preferentially occupy the external graphene surface of the carbon nanotube during the initial sorption process, while the overall adsorption of NACs included the contributions from the adsorption on the graphene surface as well as due to pore filling.

The initial steep adsorption curves may therefore be attributed to the rapid sorption onto the surface sites of MWCNTs. After the full occupancy of the surface adsorption sites had been achieved, the NACs molecules then sorbed into the interstitial channel spaces which was considered to be the rate-controlling process since NACs would have to move from the exterior surface to the inner surface of the pores of the MWCNTs to achieve equilibrium. Pore filling for activated carbon generally has a long residence time and adsorption takes, for example, about $6 \mathrm{~h}$ for nitrobenzene [15]. In contrast, the equilibrium time for NACs sorption onto CNTs is 


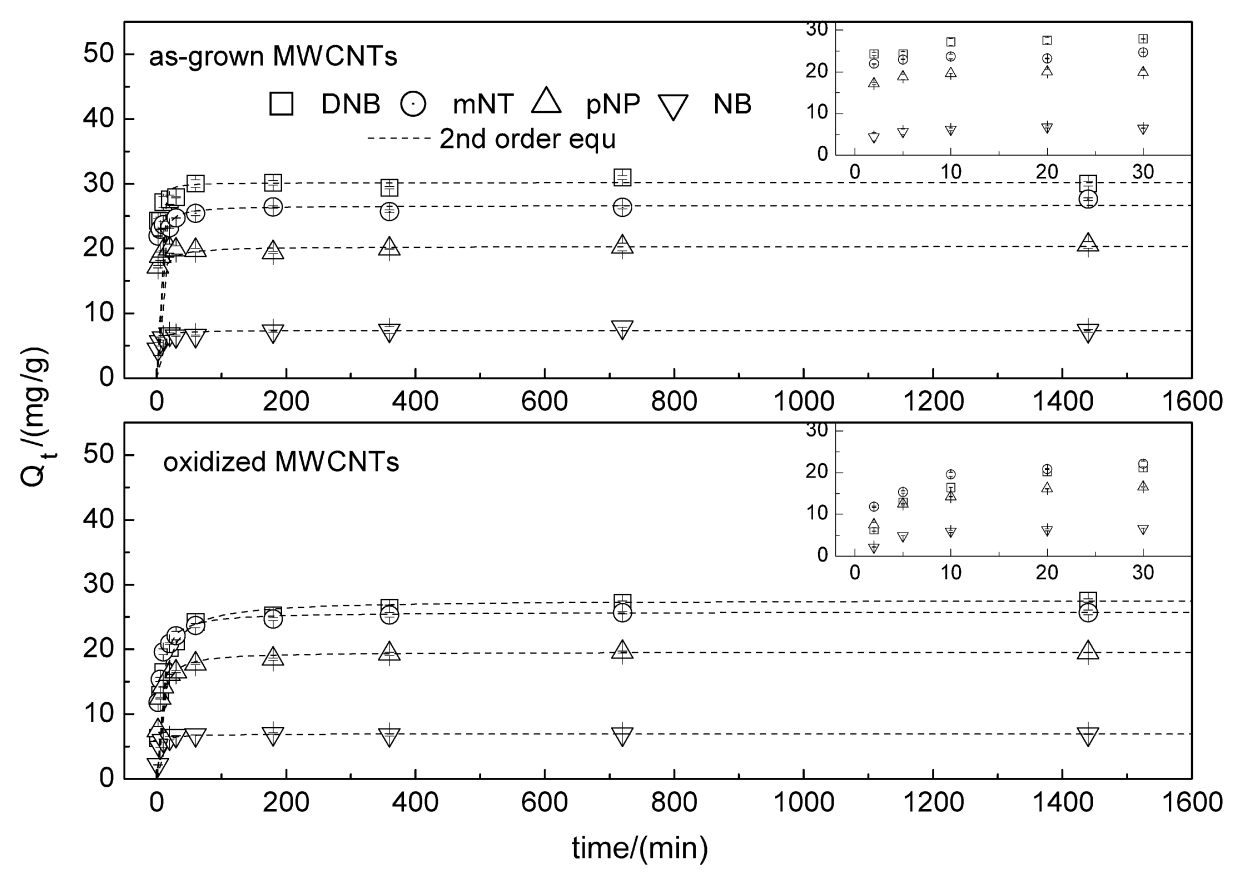

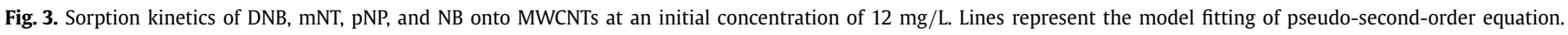

Table 3

Kinetic parameters of pseudo-second-order model fitting for the NAC adsorption onto MWCNTs.

\begin{tabular}{lllll}
\hline MWCNTs & NACs & $\begin{array}{l}v_{0} \\
{[\mathrm{mg} /(\mathrm{g} \min )]}\end{array}$ & $\begin{array}{l}k_{2} \\
{[\mathrm{~g} /(\mathrm{mg} \min )]}\end{array}$ & $R^{2}$ \\
\hline As grown & DNB & 44.84 & 0.0491 & 0.9996 \\
& $\mathrm{mNT}$ & 13.12 & 0.0185 & 0.9999 \\
& $\mathrm{pNP}$ & 8.38 & 0.0203 & 0.9995 \\
& $\mathrm{NB}$ & 4.31 & 0.0793 & 0.9991 \\
Oxidized & $\mathrm{DNB}$ & 2.90 & 0.0038 & 0.9998 \\
& $\mathrm{mNT}$ & 5.59 & 0.0084 & 0.9999 \\
& $\mathrm{pNP}$ & 3.98 & 0.0103 & 0.9999 \\
& $\mathrm{NB}$ & 3.13 & 0.0646 & 0.9999 \\
\hline
\end{tabular}

comparatively short, suggesting that the contribution to the overall sorption due to a pore filling mechanism is negligible.

While four kinetic models, pseudo-second-order model, parabolic diffusion model, modified Freundlich model, and Elovich model, were fit to the experimental data, only the pseudo-secondorder model fit the sorption kinetics well with correlation coefficient consistently $>0.999$ for all four NACs considered (Table 3 ). The other three models failed to described the experimental data well, as indicated by consistently lower $R^{2}<0.9$ (data not shown), implying that the adsorption kinetics of the NACs followed a pseudo-second-order rate law.

As shown in Table 3 , the rate constant $\left(k_{2}\right)$ for NB, on both asgrown and oxidized MWCNTs, was the largest among all four NACs considered, indicating that the sorption of NB on MWCNTs was the fastest process. This may be attributed to the smaller molar volume $\left(V_{s}\right)$ and Van der Waals square $\left(S_{V d w}\right)$ (Table 1$)$ for NB compared to the other NACs studied, since it is well known that smaller molecules are adsorbed faster than bigger ones [18].

The initial adsorption rate $\left(v_{0}\right)$ and the adsorption rate constant $\left(k_{2}\right)$ of the oxidized MWCNTs were both smaller than those of the as-grown MWCNTs, suggesting that the sorption of NACs onto asgrown MWCNTs was faster than that onto the oxidized MWCNTs (Table 3). The difference in adsorption kinetics between as-grown and oxidized MWCNTs may be due to the changes in the surface chemistry of MWCNTs after nitric acid oxidization. After oxidization, hydrophilic oxygen-containing groups, including carboxylic, carbonyl, and hydroxyl groups, were introduced to the outmost surface and defect sites of MWCNTs. The addition of theses groups results in a more negatively charged MWCNT surface due to deprotonation of carboxylic groups at the equilibrium sorption $\mathrm{pH}$ of 6 , since at this $\mathrm{pH}$ the adsorption of water is more energetically favorable relative to the sorption of NACs and thus the adsorption of NACs is more difficult.

\subsection{Sorption isotherm of NACS}

Fig. 4 presents the sorption isotherms of DNB, mNT, pNP, and NB on as-grown and oxidized MWCNTs at different temperatures. Langmuir and Freundlich models fit the sorption isotherms well (Table 4 ), and the values of $1 / n$ were generally lower than 0.5 (Table 4), consistent with the adsorption onto other carbon materials such as activated carbon [27,28] and black carbon [29,30]. Three main mechanisms have been proposed for the sorption of nondissociable aromatic compounds onto carbon materials, (1) $\pi-\pi$ dispersion interaction, (2) hydrogen bonding [12], and (3) electron donor-acceptor complex formation [13]. Of the three proposed mechanisms, $\pi-\pi$ dispersion interaction is the most widely accepted mechanism for the adsorption of aromatic compounds on activated carbon $[11,14]$. However, in this study, the $\pi-\pi$ donoracceptor complex mechanism was proposed to better explain the observed results [7]. By comparing the $Q_{m}$ of NACs adsorbed to as-grown and oxidized MWCNTs at the same temperature, $Q_{m}$ followed the order $\mathrm{DNB}>\mathrm{mNT}>\mathrm{pNP}>\mathrm{NB}$. In addition to the molecular size of the NACs, the number of nitro groups on the NACs may be another factor affecting or controlling the sorption affinity of NACs to MWCNTs. NACs are highly polar and often act as strong electron acceptors when interacting with adsorbentcontaining structures with high electron polarization or electron donors such as oxygen-containing groups. The graphene surface of MWCNTs is expected to be highly polarizable and is likely to act as an electron donor, when in contact with strong electron acceptors such as NACs. Acceptor strength of the NAC depends on the electron-withdrawing ability and the number of substitutes on the NAC. Among the four NACs studied, DNB had more $-\mathrm{NO}_{2}$ groups than any others, so its acceptor strength would be stronger and consequentially DNB should have a greater sorption affinity 


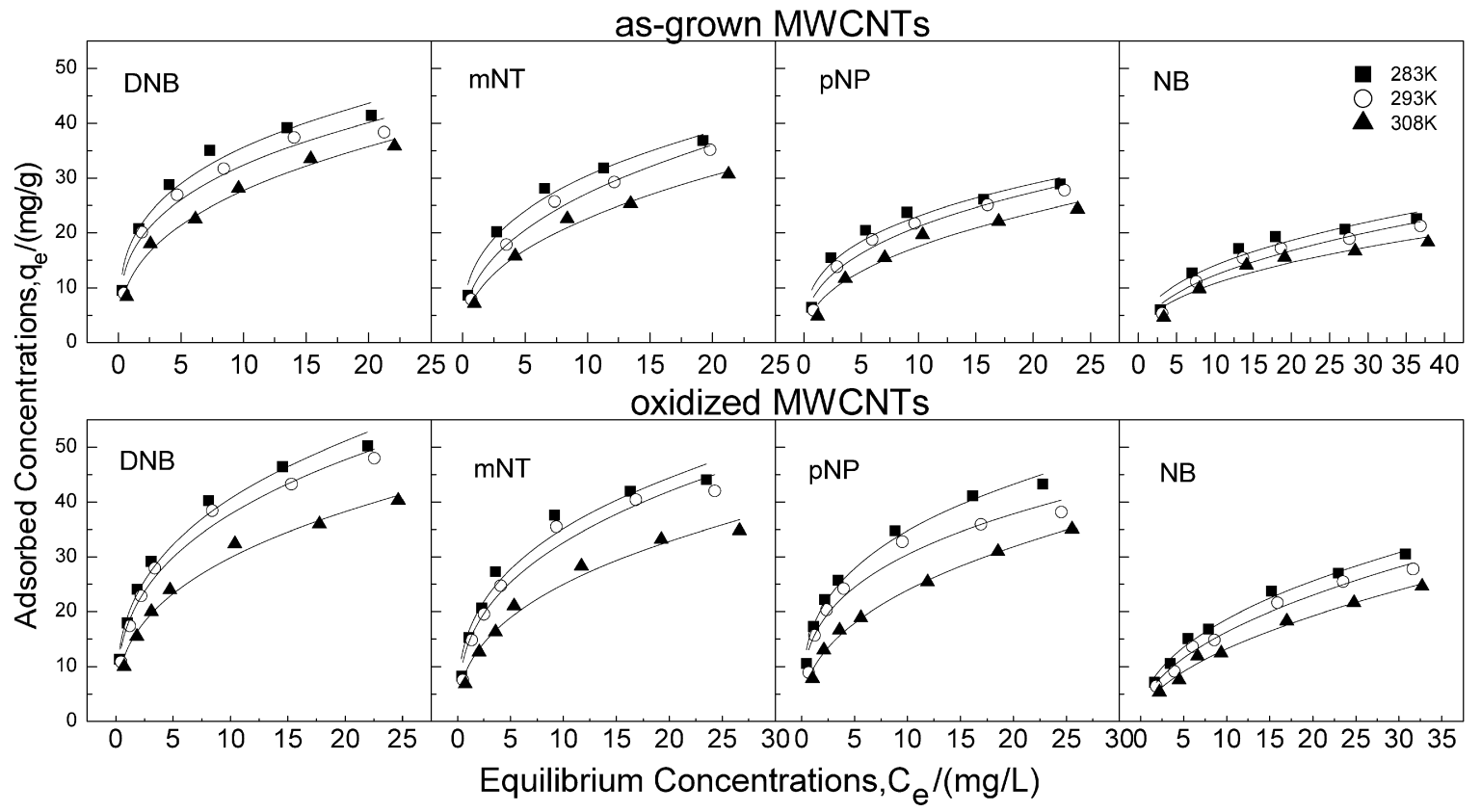

Fig. 4. Sorption isotherms of DNB, mNT, pNP, and NB onto MWCNTs at ( $\mathbf{\square}) 283 \mathrm{~K},(\bigcirc) 293 \mathrm{~K}$, and ( $\mathbf{\Delta}) 308 \mathrm{~K}$. Lines represent the model fitting of Freundlich equation.

Table 4

Coefficients of Langmuir and Freundlich model fittings for sorption of NACs on as-grown and oxidized MWCNTs at different temperatures.

\begin{tabular}{|c|c|c|c|c|c|c|c|c|c|}
\hline \multirow[t]{2}{*}{ NACs } & \multirow{2}{*}{$\begin{array}{l}\text { Temperature } \\
\text { (K) }\end{array}$} & \multicolumn{4}{|c|}{ Langmuir model } & \multicolumn{4}{|c|}{ Freundlich model } \\
\hline & & $\begin{array}{l}Q_{m^{c}} \\
(\mathrm{mg} / \mathrm{g})\end{array}$ & $\begin{array}{l}Q_{\mathrm{m}-S A} \\
\left(\mathrm{mg} / \mathrm{m}^{2}\right)\end{array}$ & $\begin{array}{l}K_{\mathrm{L}}^{\mathrm{c}} \\
(\mathrm{mg} / \mathrm{L})\end{array}$ & $R^{2}$ & $\begin{array}{l}K_{\mathrm{F}}{ }^{\mathrm{c}} \\
(\mathrm{mg} / \mathrm{g}) \\
(\mathrm{L} / \mathrm{mg})^{1 / n}\end{array}$ & $\begin{array}{l}K_{\mathrm{F}-\mathrm{SA}} \\
\left(\mathrm{mg} / \mathrm{m}^{2}\right) \\
(\mathrm{L} / \mathrm{mg})^{1 / n}\end{array}$ & $1 / n^{\mathrm{c}}$ & $R^{2}$ \\
\hline \multirow[t]{6}{*}{ DNB } & $283^{\mathrm{a}}$ & $44.0 \pm 2.04$ & 0.275 & $1.76 \pm 0.35$ & 0.977 & $18.1 \pm 1.45$ & 0.113 & $0.30 \pm 0.033$ & 0.969 \\
\hline & $293^{\mathrm{a}}$ & $41.4 \pm 1.69$ & 0.259 & $2.07 \pm 0.35$ & 0.983 & $15.8 \pm 1.48$ & 0.099 & $0.31 \pm 0.038$ & 0.963 \\
\hline & $308^{\mathrm{a}}$ & $40.4 \pm 2.87$ & 0.253 & $3.64 \pm 0.90$ & 0.967 & $11.8 \pm 0.91$ & 0.074 & $0.37 \pm 0.030$ & 0.984 \\
\hline & $283^{b}$ & $53.1 \pm 2.43$ & 0.194 & $2.14 \pm 0.35$ & 0.981 & $18.9 \pm 0.98$ & 0.069 & $0.33 \pm 0.021$ & 0.987 \\
\hline & $293^{b}$ & $48.7 \pm 1.52$ & 0.178 & $2.72 \pm 0.35$ & 0.954 & $16.0 \pm 0.33$ & 0.058 & $0.34 \pm 0.008$ & 0.993 \\
\hline & $308^{b}$ & $44.2 \pm 1.92$ & 0.161 & $3.57 \pm 0.50$ & 0.986 & $12.7 \pm 0.76$ & 0.046 & $0.37 \pm 0.023$ & 0.987 \\
\hline \multirow[t]{6}{*}{$\mathrm{mNT}$} & $283^{\mathrm{a}}$ & $43.9 \pm 2.52$ & 0.273 & $2.49 \pm 0.61$ & 0.977 & $13.9 \pm 1.14$ & 0.087 & $0.34 \pm 0.034$ & 0.983 \\
\hline & $293^{\mathrm{a}}$ & $41.4 \pm 3.04$ & 0.259 & $4.36 \pm 0.99$ & 0.982 & $10.9 \pm 0.88$ & 0.067 & $0.41 \pm 0.033$ & 0.988 \\
\hline & $308^{a}$ & $37.3 \pm 2.60$ & 0.234 & $5.46 \pm 1.11$ & 0.985 & $8.4 \pm 0.82$ & 0.053 & $0.43 \pm 0.037$ & 0.986 \\
\hline & $283^{b}$ & $48.5 \pm 1.66$ & 0.177 & $2.65 \pm 0.32$ & 0.991 & $15.9 \pm 1.33$ & 0.058 & $0.35 \pm 0.033$ & 0.973 \\
\hline & $293^{\mathrm{b}}$ & $47.8 \pm 1.35$ & 0.175 & $3.35 \pm 0.31$ & 0.995 & $13.5 \pm 1.21$ & 0.049 & $0.39 \pm 0.034$ & 0.992 \\
\hline & $308^{b}$ & $41.0 \pm 1.43$ & 0.150 & $4.92 \pm 0.52$ & 0.993 & $9.8 \pm 0.80$ & 0.036 & $0.41 \pm 0.030$ & 0.983 \\
\hline \multirow[t]{6}{*}{ pNP } & $283^{\mathrm{a}}$ & $32.2 \pm 0.83$ & 0.201 & $2.59 \pm 0.27$ & 0.993 & $10.9 \pm 1.30$ & 0.068 & $0.33 \pm 0.047$ & 0.948 \\
\hline & $293^{\mathrm{a}}$ & $31.7 \pm 0.91$ & 0.198 & $3.95 \pm 0.38$ & 0.995 & $8.9 \pm 0.97$ & 0.055 & $0.38 \pm 0.042$ & 0.969 \\
\hline & $308^{\mathrm{a}}$ & $30.6 \pm 0.99$ & 0.192 & $6.25 \pm 0.57$ & 0.996 & $6.4 \pm 0.93$ & 0.040 & $0.44 \pm 0.054$ & 0.962 \\
\hline & $283^{\mathrm{b}}$ & $45.8 \pm 1.87$ & 0.167 & $2.21 \pm 0.32$ & 0.984 & $14.8 \pm 1.33$ & 0.054 & $0.36 \pm 0.036$ & 0.964 \\
\hline & $293^{b}$ & $41.1 \pm 1.59$ & 0.150 & $4.00 \pm 0.44$ & 0.994 & $9.7 \pm 0.55$ & 0.035 & $0.45 \pm 0.024$ & 0.978 \\
\hline & $308^{b}$ & $39.9 \pm 2.30$ & 0.146 & $5.27 \pm 0.88$ & 0.982 & $8.7 \pm 0.62$ & 0.032 & $0.43 \pm 0.026$ & 0.988 \\
\hline \multirow[t]{6}{*}{ NB } & $283^{\mathrm{a}}$ & $28.3 \pm 1.06$ & 0.177 & $8.97 \pm 0.99$ & 0.992 & $5.5 \pm 1.09$ & 0.034 & $0.41 \pm 0.064$ & 0.936 \\
\hline & $293^{\mathrm{a}}$ & $27.8 \pm 1.01$ & 0.174 & $11.63 \pm 1.12$ & 0.995 & $4.3 \pm 0.79$ & 0.027 & $0.45 \pm 0.058$ & 0.956 \\
\hline & $308^{b}$ & $24.0 \pm 1.41$ & 0.151 & $11.40 \pm 1.80$ & 0.986 & $3.9 \pm 0.91$ & 0.024 & $0.44 \pm 0.075$ & 0.927 \\
\hline & $283^{b}$ & $38.1 \pm 1.67$ & 0.139 & $8.85 \pm 1.00$ & 0.992 & $5.2 \pm 0.34$ & 0.019 & $0.47 \pm 0.022$ & 0.990 \\
\hline & $293^{\mathrm{b}}$ & $37.5 \pm 2.32$ & 0.137 & $11.45 \pm 1.68$ & 0.988 & $4.1 \pm 0.24$ & 0.015 & $0.50 \pm 0.019$ & 0.989 \\
\hline & $308^{\mathrm{b}}$ & $35.1 \pm 2.49$ & 0.128 & $15.02 \pm 2.32$ & 0.989 & $3.8 \pm 0.31$ & 0.014 & $0.54 \pm 0.027$ & 0.992 \\
\hline
\end{tabular}

$Q_{\mathrm{m}-\mathrm{SA}}$ and $K_{\mathrm{F}-\mathrm{SA}}$ were coefficients of $Q_{\mathrm{m}}$ and $K_{\mathrm{F}}$ normalized by surface area.

a As-grown MWCNTs.

b Oxidized MWCNTs.

c Mean \pm standard deviation.

than the other NACs considered. For theses reasons, $\pi-\pi$ electron donor acceptor interactions may be the most important interaction between NACs and MWCNTs where the number of nitro groups becomes a significant factor affecting and controlling the sorption.

Since nitric acid oxidation removed a large amount of impurities, including amorphous carbon and metal particles allowing the formation of bundles, the surface area and pore volumes of MWC-
NTs were consequentially increased by $\backsim 70 \%$ compared to the asgrown MWCNTs (Table 2), and the sorption capacity of MWCNTs ( $Q_{\mathrm{m}}$ and $K_{\mathrm{F}}$ ) was increased for all the NACs studied (Table 4). In order to distinguish the effect of surface area and pore volume on the sorption of NACs from that of oxygen-containing groups, the sorption capacity ( $Q_{\mathrm{m}-\mathrm{SA}}$ and $K_{\mathrm{F}-\mathrm{SA}}$ ) was normalized on the basis of surface area, and $Q_{\mathrm{m}-\mathrm{SA}}$ and $K_{\mathrm{F}-\mathrm{SA}}$ compared between the ni- 


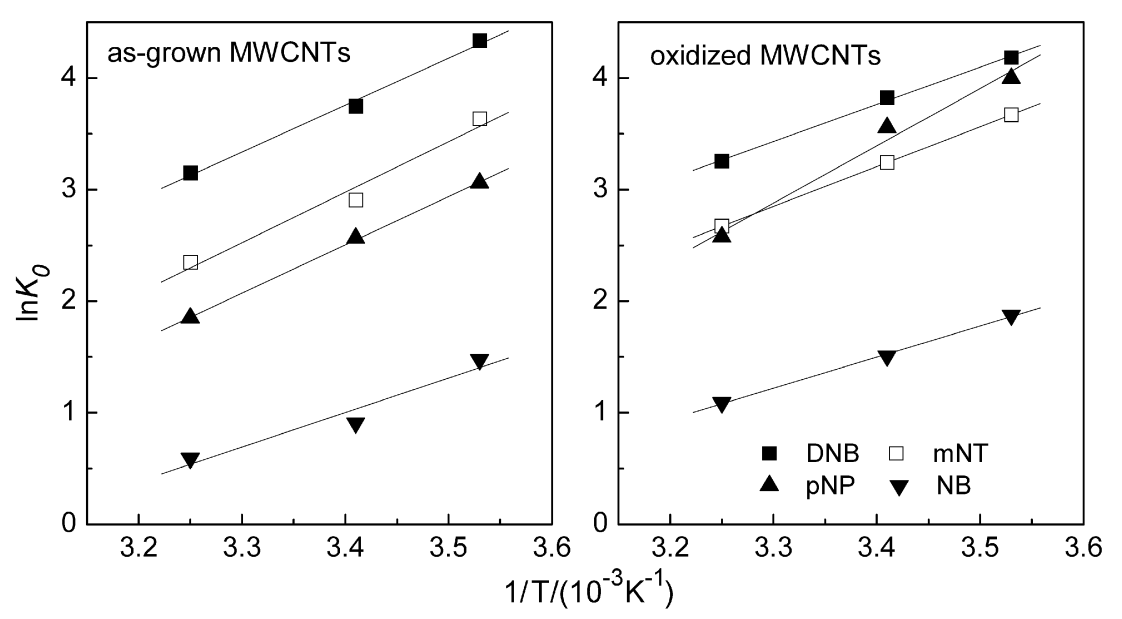

Fig. 5. Plots of $\ln K_{0}$ versus $1 / T$ of sorption of DNB, mNT, pNP, and NB on MWCNTs.

tric acid-oxidized and as-grown MWCNTs. This comparison showed that $Q_{\mathrm{m}-\mathrm{SA}}$ and $K_{\mathrm{F}-\mathrm{SA}}$ of the nitric acid-oxidized MWCNTs were consistently lower than those of as-grown MWCNTs for all the NACs studied (Table 4). The existence of oxygen functional groups on the nitric-oxidized MWCNTs, which increased the hydrophilicity of MWCNTs, had already been identified (Fig. 2). Numerous studies demonstrated that the oxygen functional groups depressed the sorption of organic chemicals on carbon materials $[27,30]$ via water adsorption, dispersive/repulsive interactions, and hydrogen bonding. A recent study [8] also revealed that incorporated surface oxides created polar regions that reduced the surface area available for naphthalene sorption, and as little as a $10 \%$ increase in surface oxygen concentration resulted in a decrease of the maximum adsorption capacity of naphthalene by roughly $70 \%$.

\subsection{Thermodynamic analysis}

Thermodynamic parameters provide additional in-depth information regarding the inherent energetic changes involved during sorption. To assess the thermodynamic parameters, the sorption isotherms of DNB, mNT, pNP, and NB onto MWCNTs were measured at 283,293 , and $308 \mathrm{~K}$ and the changes in thermodynamic parameters of free energy of sorption $(\Delta G)$, enthalpy $(\Delta H)$, and entropy $(\Delta S)$ were calculated from the variation of the thermodynamic equilibrium constant $K_{0}$ with the change in temperature. The constant $K_{0}$ for the adsorption reaction can be defined as

$K_{0}=\frac{a_{\mathrm{s}}}{a_{\mathrm{e}}}=\frac{v_{\mathrm{s}}}{v_{\mathrm{e}}} \frac{q_{\mathrm{e}}}{C_{\mathrm{e}}}$,

where $a_{\mathrm{s}}$ is the activity of adsorbed NACs, $a_{\mathrm{e}}$ is the activity of NACs in solution at equilibrium, $q_{\mathrm{e}}$ is the surface concentration of NACs on MWCNTs (mg/g), $C_{\mathrm{e}}$ is the concentration of NACs at equilibrium solution $(\mathrm{mg} / \mathrm{L}), v_{\mathrm{s}}$ is the activity coefficient of the adsorbed NACs, and $v_{\mathrm{e}}$ is the activity coefficient of NACs in solution. As the concentration of NACs in the solution approaches zero, the activity coefficient approaches unity, reducing Eq. (9) to the following form:

$K_{0}=\frac{a_{\mathrm{s}}}{a_{\mathrm{e}}}=\frac{q_{\mathrm{e}}}{C_{\mathrm{e}}}$.

Values of $K_{0}$ were therefore obtained by plotting $\ln \left(q_{\mathrm{e}} / C_{\mathrm{e}}\right)$ versus $q_{\mathrm{e}}$, and extrapolating $q_{\mathrm{e}}$ to zero. The straight line obtained was fit using least-squares analysis where the intercept of the vertical axis was $\ln K_{0}$.

Free energy changes $\Delta G$ for any interaction can be calculated using the relationship

$\Delta G=-R T \ln K_{0}$,
Table 5

Thermodynamics parameters of NACs sorbed on MWCNTs.

\begin{tabular}{|c|c|c|c|c|c|c|}
\hline \multirow[t]{2}{*}{ MWCNTs } & \multirow[t]{2}{*}{ NACs } & \multicolumn{3}{|c|}{$\Delta G(\mathrm{~kJ} / \mathrm{mol})$} & \multirow{2}{*}{$\begin{array}{l}\Delta H \\
(\mathrm{~kJ} / \mathrm{mol})\end{array}$} & \multirow{2}{*}{$\begin{array}{l}\Delta S \\
{[\mathrm{~J} /(\mathrm{mol} \mathrm{K})}\end{array}$} \\
\hline & & $283 \mathrm{~K}$ & $293 \mathrm{~K}$ & $308 \mathrm{~K}$ & & \\
\hline \multirow[t]{4}{*}{ As grown } & DNB & -10.57 & -9.59 & -8.07 & -34.10 & -84.64 \\
\hline & $\mathrm{mNT}$ & -8.85 & -7.44 & -6.01 & -36.71 & -100.02 \\
\hline & pNP & -7.68 & -6.57 & -4.74 & -37.69 & -107.00 \\
\hline & NB & -3.60 & -2.33 & -1.52 & -25.05 & -76.82 \\
\hline \multirow[t]{4}{*}{ Oxidized } & DNB & -10.19 & -9.80 & -8.33 & -27.08 & -60.75 \\
\hline & $\mathrm{mNT}$ & -8.64 & -7.63 & -6.29 & -28.99 & -71.91 \\
\hline & pNP & -9.74 & -8.67 & -6.27 & -41.77 & -113.80 \\
\hline & NB & -4.57 & -3.67 & -2.65 & -22.64 & -64.49 \\
\hline
\end{tabular}

where $R$ is the universal gas constant and $T$ is the temperature in Kelvin. Rearrangement and substitution give

$R T \ln K_{0}=T \Delta S-\Delta H$,

$\ln K_{0}=-\Delta H / R T+\Delta S / R$,

where Eq. (12) describes how the equilibrium constant $K_{0}$ varies with absolute temperature $T$ for an equilibrium system and Eq. (13) predicts a linear plot of $\ln K_{0}$ versus $1 / T$ for reversible sorption of NACs on MWCNTs. The slope of Eq. (13) is $-\Delta H / R$, and the intercept is $\Delta S / R$ (Fig. 5) [31]. $\Delta G(\mathrm{~kJ} / \mathrm{mol}), \Delta H(\mathrm{~kJ} / \mathrm{mol})$, and $\Delta S[\mathrm{~J} /(\mathrm{mol} \mathrm{K})]$ values are given in Table 5 .

The $\Delta G$ values were negative for all four NACs at all three temperatures, confirming that the sorption of NACs onto MWCNTs was spontaneous and thermodynamically favorable. The observed negative $\Delta H$ suggested an exothermic sorption, which was supported by the observation that sorption of NACs onto MWCNTs decreased with increasing sorption temperature (Fig. 4). Kara et al. [32] suggested that the $\Delta H$ of physisorption was less than $40 \mathrm{~kJ} / \mathrm{mol}$, implying the sorption of NACs onto MWCNTs was a physisorption process [33] except for the sorption of pNP on the oxidized MWCNTs $(\Delta H=41.77 \mathrm{~kJ} / \mathrm{mol})$. The negative $\Delta S$ indicated decreased randomness at the solid-liquid interface during sorption as might be expected from acceptor donor complex formation.

The values of $\Delta H$ and $\Delta S$, for the sorption of NACs onto oxidized MWCNTs and as-grown MWCNTs, decreased after nitric acid oxidation except for pNP. The value of $\Delta H$ for pNP increased remarkably after nitric acid oxidation. The significantly higher thermodynamics adsorption constants observed for pNP in the oxidized MWCNTs may be due to specific interactions between pNP and the oxidized MWCNTs. One such specific interaction is the formation of hydrogen bonds and pNP is the only dissociable compound among the four NACs studied that has a hydroxy functional 
group $(-\mathrm{OH})$ capable of forming H-bonds with the surface oxygen groups of the MWCNTs. Some authors have also identified the existence of $\mathrm{H}$-bonds between the phenol and carboxyl groups of activated carbon, although water can compete with phenol [14,34]. It is well known that adsorption of phenolic compounds to carbon materials is partly physical and partly chemical [11]. While physisorbed phenol compounds can be desorbed by using different solvents or by heat treatments in inert atmosphere, the chemisorbed compounds cannot be desorbed, even at high temperatures, due to the strong chemical bond between phenolic compounds and the carbon surface formed. In general, since the $\Delta H$ of a chemical reaction is much larger than the $\Delta H$ for a physical reaction the large change of enthalpy for sorption of pNP onto oxidized MWCNTs is most likely attributable to chemical sorption of pNP via hydrogen bonding.

\section{Summary}

The kinetics and thermodynamics of NAC sorption onto asgrown and nitric acid-oxidized MWCNTs were investigated. The sorption followed a pseudo-second-order kinetics law. The sorption of NACs onto MWCNTs was exothermic and spontaneous where both the Langmuir and the Freundlich models described the sorption data well. Molecule size and the number of nitro groups of the NACs were the main factors that affected sorption kinetics and the equilibrium. Nitric acid oxidation increased the surface area and pore volume of the MWCNTs and introduced oxygen functional groups to MWCNTs which depressed the sorption rate and sorption capacity of NACs onto MWCNTs.

\section{References}

[1] R.Q. Long, R.T. Yang, J. Am. Chem. Soc. 123 (2001) 2058.

[2] X. Peng, Y. Li, Z. Luan, Z. Di, H. Wang, B. Tian, Z. Jia, Chem. Phys. Lett. 376 (2003) 154
[3] C. Lu, Y.L. Chung, K.F. Chang, Water Res. 39 (2005) 1183.

[4] K. Yang, L. Zhu, B. Xing, Environ. Sci. Technol. 40 (2006) 1855.

[5] C.J.M. Chin, L.C. Shih, H.J. Tsai, T.K. Liu, Carbon 45 (2007) 1254.

[6] Q. Liao, J. Sun, L. Gao, Carbon 46 (2008) 553.

[7] W. Chen, L. Duan, D. Zhu, Environ. Sci. Technol. 41 (2007) 8295.

[8] H.H. Cho, B.A. Smith, J.D. Wnuk, D.H. Fairbrother, W.P. Ball, Environ. Sci. Technol. 42 (2008) 2899.

[9] V. Datsyuk, M. Kalyva, K. Papagelis, J. Parthenios, D. Tasis, A. Siokou, I. Kallitsis, C. Galiotis, Carbon 46 (2008) 833.

[10] L.R. Radovic, I.F. Silva, J.I. Ume, J.A. Menéndez, C. Leon, A.W. Scaroni, Carbon 35 (1997) 1339.

[11] C. Moreno-Castilla, Carbon 42 (2004) 83.

[12] R.W. Coughlin, F.S. Ezra, Environ. Sci. Technol. 2 (1968) 291.

[13] J.A. Mattson, H.B. Mark, M.D. Malbin, W.J. Weber, J.C. Crittenden, J. Colloid Interface Sci. 31 (1969) 116.

[14] S. Haydar, M.A. Ferro-Garcia, J. Rivera-Utrilla, J.P. Joly, Carbon 41 (2003) 387.

[15] F. Villacańas, M.F.R. Pereira, J.J.M. Órfăo, J.L. Figueiredo, J. Colloid Interface Sci. 293 (2006) 128.

[16] S.B. Haderlein, K.W. Weissmahr, R.P. Schwarzenbach, Environ. Sci. Technol. 30 (1996) 612.

[17] S.B. Haderlein, R.P. Schwarzenbach, Environ. Sci. Technol. 27 (1993) 316.

[18] C. Lu, Y.L. Chung, K.F. Chang, J. Hazard. Mater. 138 (2006) 304.

[19] C.H. Wu, J. Hazard. Mater. 144 (2007) 93.

[20] Y.S. Ho, G. McKay, Water Res. 34 (2000) 735.

[21] Z. Aksu, A. Tatl, Ö. Tunc, Chem. Eng. J. 142 (2008) 23.

[22] S. Azizian, J. Colloid Interface Sci. 276 (2004) 47.

[23] S. Azizian, B. Yahyaei, J. Colloid Interface Sci. 299 (2006) 112.

[24] X. Yang, B. Al-Duri, J. Colloid Interface Sci. 287 (2005) 25.

[25] Z. Li, Langmuir 15 (1999) 6438.

[26] S. Agnihotri, J.P.B. Mota, M. Rostam Abadi, M.J. Rood, Carbon 44 (2006) 2376.

[27] M. Franz, H.A. Arafat, N.G. Pinto, Carbon 38 (2000) 1807.

[28] P.A.M. Mourão, P.J.M. Carrott, M.M.L. Ribeiro Carrott, Carbon 44 (2006) 2422.

[29] D. Zhu, S. Kwon, J.J. Pignatello, Environ. Sci. Technol. 39 (2005) 3990.

[30] D. Zhu, J.J. Pignatello, Environ. Sci. Technol. 39 (2005) 2033.

[31] E.A. Ghabbour, G. Davies, M.E. Goodwillie, K. O’Donaughy, T.L. Smith, Environ. Sci. Technol. 38 (2004) 3338.

[32] M. Kara, H. Yuzer, E. Sabah, M.S. Celik, Water Res. 37 (2003) 224.

[33] B. von Oepen, W. Kordel, W. Klein, Chemosphere 22 (1991) 285.

[34] O.P. Mahajan, C. Moreno-Castilla, P.L. Walker, Sep. Sci. Technol. 15 (1980) 1733. 